\title{
El significado de la pregunta y la respuesta en el contexto de la vida: problematización del conocimiento
}

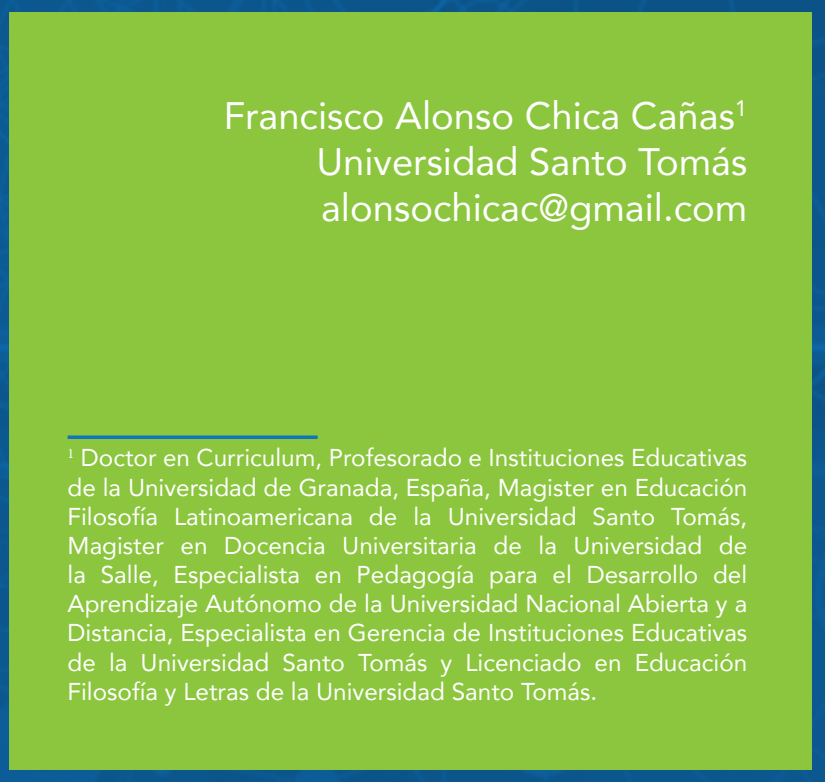

Cómo citar este artículo: Chica, F. A. (2016). El significado de la pregunta y la respuesta en el contexto de la vida: problematización del conocimiento. Revista Virtualmente, 4(2), 22-41. 


\section{RESUMEN}

La reflexión que plantea este artículo tiene como objetivo analizar la forma en que la pregunta y la respuesta se encuentra arraigada en el diario vivir de los hombres y mujeres y en ese mismo sentido, identificar su actuar en torno al mundo, que se constituye en un referente contextual de significado al involucrar los intereses de los individuos de índole existencial y vitalista, respecto de los intereses que persigue la sociedad y el conocimiento científico. Las preguntas y las repuestas que se formula el aprendiente deben contribuir al desarrollo de la autonomía de mayoría de edad, esto en concordancia con la toma las decisiones con madurez, con prudencia, con justicia, con equidad e igualdad, siendo altamente significativas en favor de la vida y la conservación de la vida como tal.

Palabras clave: pregunta-respuesta, aprendiente, constitución existenciaria, mayoría de edad. 


\section{The meaning of the question and the answer in the context of life: problematization of knowledge}

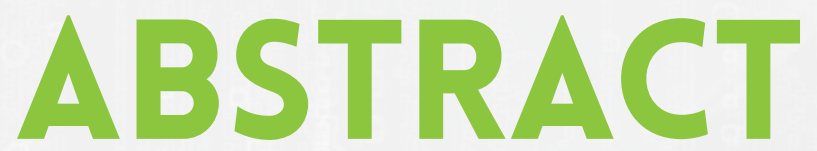

The reflection that this article raises aims to analyze the way in which the question and the answer are rooted in the daily life of men and women, and in that same sense, to identify its course of action around the world, that constitutes in a contextual reference of meaning by involving the interests proceeding from individuals of existential and vitalist nature, with respect to the interests that are pursued by society and scientific knowledge. The questions and the answers posed by the learner should contribute to the development of adulthood autonomy, this in accordance with making decision with maturity, with prudence, with justice, with equity and equality, being highly significant in favor of life and the preservation of life as such.

Keywords: question-answer, learner, existential constitution, adulthood. 


\section{Signification de la question- réponse dans le contexte de la vie:problématisation de la connaissance}

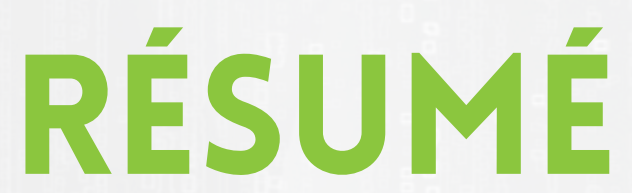

Cet article propose une réflexion ayant pour objectif d'analyser la façon dont la questionréponse est enracinée dans le quotidien des êtres humains pour donner un sens à leurs actions et constituant un référant contextuel de significations impliquant les intérêts existentiels et vitaux des individus par rapport aux intérêts poursuivis par la société et la connaissance scientifique. Les questions et les réponses formulées par l'apprenant doivent contribuer au développement de l'autonomie en concordance avec la prise de décisions responsables, la prudence, la justice, l'équité et l'égalité tout en étant hautement significatives pour la vie et sa conservation.

Mots clefs: question - réponse, apprenant, constitution existentielle, majorité. 


\section{O significado da pergunta e da resposta no contexto da vida:problematização do conhecimento}

\section{RESUMO}

A reflexão sugerida por este artigo tem por objetivo analisar a forma como a pergunta e a resposta estao enraizadas no cotidiano de homens e mulheres e nesse sentido, identificar as suas ações ao redor do mundo, que se torna um significado contextual referente a envolver os interesses dos indivíduos de indole existencial e vitalista, respeito aos interesses prosseguidos pela sociedade e o conhecimento científico. As perguntas e as respostas formuladas pelos alunos devem contribuir para o desenvolvimento da autonomia da maioria da idade, que de acordo a tomada de decisão com maturidade, com sabedoria, com justiça, equidade e igualdade, sendo altamente significativa a favor da vida e da preservação da vida como tal.

\footnotetext{
Palavras-chave: pergunta-resposta, aprendiz, constituição existencial, maioria da idade.
} 


\section{Introducción}

Sedano (2002) plantea la pregunta de ¿para qué educar en tiempos convulsionados y críticos? Por tanto, se requiere una pedagogía inspiradora de la vida que plantee preguntas y respuestas hacia la construcción de la perfección humana según la metodología de ver, jugar y actuar, con el propósito de formar la conducta en búsqueda de una autodeterminación comprometida con el colectivo o la comunidad que se encarga de problematizar el conocimiento para que el individuo se promueva a sí mismo y con el otro, siendo la vida el punto de partida de la pregunta respecto de la existencia humana.

El aprendiente como un ser ahí arrojado en el mundo plantea una serie de preguntas y respuestas relacionadas con la propia existencia, lo cual implica establecer lazos de interacción cognitivos, emotivos, afectivos y amorosos para encontrarse con sí mismo y con el Otro, con el fin de comprenderse e interpretarse como imperativo existencial para introducirse en la incertidumbre y certeza de la sociedad del conocimiento en la desconstrucción y construcción de la realidad. Esto significa asumir la pregunta y la respuesta como un mundo de posibilidades para proyectarse en medio de la biografía a fin de construir conocimiento a partir de una inteligencia encarnada que asume la comunicación como una instancia de apertura en la palabra para encontrarse y curarse en medio del peligro existenciario de la vida.

Del mismo modo, el papel existenciario tiene razón en el lenguaje en cuanto comparte las experiencias personales y de las otras personas en el plano de las racionalidades del mundo cotidiano y del mundo de la vida. Esto implica optar por ropajes cognitivos que son el producto de las preguntas y las respuestas problematizadoras sobre cuestiones que autodeterminan y autoobligan a autodecidirse sobre los dilemas del conocimiento de orden reflexivo para la realización de la plenitud del propio ser en la existencia. Lo anterior con el fin de personalizarse y comprometerse en el acto de la propia libertad, teniendo como horizonte de búsqueda de la propia autonomía de indagación en torno al bien común de aquello que está problematizado en la biografía del aprendiente. Con esto, se dejan de lado los intereses de cálculos y fines en función de una vida de autorrealización y de felicidad para alcanzar una vida buena que implica habérselas con el mundo.

\section{Pedagogía de la respuesta en fray José de Jesús Sedano González, O.P.}

Sedano (2002) profundiza en la pedagogía de la respuesta, teniendo en cuenta la formulación de preguntas, tales como:

1. Estas preguntas parten de sí mismo y de los otros que esconden una serie de interrogantes agazapados que son interminables en la vida cotidiana.

2. La pregunta forma para la libertad comprometida, porque la persona tiene una autoconvicción y autodecisión, lo cual implica formar para una libertad respecto de ver, 
juzgar y actuar. El individuo tiene que discernir a fin de comprometerse de forma crítica para educarse a sí mismo, concibiendo la autonomía como un ejercicio de confianza en la libertad.

3. Promover para la auténtica docilidad según la escucha de la experiencia de los mayores para llegar a un autocontrol y dominio de la personalidad, siendo inherente al desarrollo de una inteligencia disciplinada.

4. El sentido crítico y de discernimiento radica en la formación del juico, con el fin de poseer una visión de síntesis vital del mundo.

5. El sentido de creatividad y de iniciativa para responder de forma imaginativa a los problemas de la vida y educarse para el cambio.

6. El gran reto que enfrenta el educador es lograr una trasformación y cambio en los estudiantes, en especial cuando el mundo actual pondera el dominio de la técnica y de la razón como medio de eficiencia y eficacia para acceder a la sociedad del conocimiento global. Sin embargo, el hombre necesita de Dios para propiciar una formación desde adentro del individuo y comprometerse entrañablemente con $\mathrm{El}$, en una sociedad secularizada y plagada por la razón instrumental de la ciencia y la técnica.

Para Sedano (2002), la pedagogía de la respuesta está orientada hacia el ideal de perfección humana que se evidencia a través del acto educativo. En otras palabras, al ideal de formación le corresponde un enfoque educativo de acuerdo con la pregunta: ¿cuál es el hombre que se desea formar en la sociedad? De ahí que:

1. Sobre las diversas concepciones sobre el hombre y la vida, pueden plantearse diferentes corrientes pedagógicas.

2. Es importante tener un ideal de educación que corresponda con la realidad y que se operativo.

3. La vida concebida desde una perspectiva pedagógica le imprime fecundidad a la educación.

4. La educación es realidad cuando lo asume una comunidad educadora, dirigida hacia la vida del colectivo o del grupo, en búsqueda de los saberes, al tiempo que se convierte en un arte de formación.

5. La pedagogía dominicana concibe el ideal de la vida como una respuesta a la problemática cultural que proporciona criterios pedagógicos ante el Evangelio.

Por esta razón, la pedagogía de la pregunta y la respuesta desempeña un papel decisivo en la formación del estudiante, porque compete a la vida, a la existencia y a la ética. Entonces, la reflexión sobre el significado de la pregunta y la respuesta es determinante, puesto que tiene que ver con la inteligencia cognitiva, emocional, afectiva, social, cultural y científica de individuo y del grupo. Cuando el estudiante problematiza el conocimiento mediante un interrogatorio que forma parte de la vida, está desarrollando habilidades de pensamiento de orden superior, como la indagación, la exploración, la contemplación y el pensamiento hipotético, entre otros. Para Sedano (2002), la vida adquiere un referente problematizador, 
porque las preguntas y las repuestas conllevan un alto sentido de responsabilidad para promoverse a sí mismo, lo cual implica definirse-autodeterminarse para comprometerse consigo mismo y con los demás. Por ende, "la realidad de lo otro y de los otros (Dios y los prójimos, comunidad humana, social, eclesial...) es el punto de partida de tu conocimiento, y de tu conciencia reflexiva y decisoria, y de tu singularidad, 'principio frontal' de tu responsabilidad" (p. 222).

La pedagogía de la pregunta y la respuesta genera reflexión y cuestionamiento acerca de lo que hace en la vida, es una pregunta que no puede postergase, porque forma parte de la existencia del hombre que contribuye al crecimiento intelectual, moral y científico en torno a habérselas con el mundo. La pregunta permite descubrir aquellos filones de la vida, tan importante en la búsqueda de la felicidad, del conocimiento, de la ciencia y la tecnología. La vida es un don y una potencia para la realización perfectible de la existencia en torno al destino del hombre que se materializa en interrogatorios y en alternativas para una realización plena del individuo que interactúa ante una sociedad.

Para Sedano (2002), la vida tiene como característica un organismo vivo dotado de cualidades, "de energías propias, con una figura y una identidad tan propias que nos individualizan, nos distinguen de los demás y nos hacen una persona singular e incanjeable" (p. 183). Estas individualidades son necesarias en la especie humana, siempre y cuando se alcance una identidad del bien común en medio de la dialéctica que genera la pregunta y la respuesta. Sin embargo, la sociedad contemporánea vive una crisis de valores que atenta contra la dignidad de la persona y desdibuja la imagen de Dios, en cuanto somos capaces de testimoniar el amor y la grandeza de Dios.

\section{La pregunta y la respuesta como constitución existenciaria del aprendiente}

La constitución existenciaria del aprendiente aparece como un ser abierto al mundo en cuanto el estado de ánimo posibilita poner a flote los sentimientos para formular preguntas y respuestas como un ser ahí que se interroga por el destino, por la vida, por el Otro, por las cosas, por el mundo, por el universo, por el trabajo, por la educación, por la comida, por la vivienda, por el amor, por el afecto, por los amigos, por lo familiares, en fin, por conocer su propia existencia para sobrevivir y competir en una sociedad consumista y globalizada por un enfoque economicista.

El aprendiente está arrojado en el mundo y establece lazos de interacción para conocer e intervenir de modo cognitivo, metacognitivo, ético, social, cultural, científico, político, comunitario, y otros. Entonces, se impone la primacía del conocimiento para encontrarse consigo mismo, con el Otro y con el mundo, en el sentido de que, el ser ahí, como realidad de realidades, se constituye en un imperativo de conocer, de querer, de interpretar, de analizar, de interrogar y de comunicarse para ir descubriendo la verdad a partir de una inteligencia intuitiva y una inteligencia de pensamiento hipotético. Por tal razón,

el hombre ha entendido desde el tiempo de los inicios de la civilización humana la importancia que tiene el conocimiento para comprender la existencia natural y social, articularse adecuadamente a ella y transformarla en forma significativa para su propio beneficio. (Medina, 1997, p. 30) 
De igual manera, el aprendiente permanece en un estado abierto, lo cual conlleva, según Heidegger (2007), ver en torno, entendido como el conocimiento sensorial y el conocimiento formal para encontrarse con la verdad de la realidad a través de la incertidumbre y la certeza del conocimiento. En palabras heideggerianas, sería encontrarse para ser golpeado por la realidad, al tiempo que la persona es herida, por estar abierto al mundo, lo cual implica, desde la perspectiva de Varela (2000), manipular las cosas mediante una percepción motora (dimensión local) y la inteligencia encarnada en el sujeto (dimensión de la persona) que produce un conocimiento cognitivo resultado de una modificación para que emerja un nuevo concepto. Es decir, "el objeto surge como fruto de nuestra actividad, por lo tanto, tanto el objeto como la persona están co-emergiendo, co-surgiendo" (p. 241).

La existencia tiene como referencia al Otro, quien, por medio de las pasiones y los sentimientos, genera un conocimiento referencial de voluntad. La persona está abierta al mundo, pero, si no tiene voluntad de querer hacer las cosas, no afectará a las otras personas y el mundo como tal. Entonces, el aprendiente asume su existencia bajo una perspectiva de voluntad, con el fin de crear representaciones mentales del mundo para participar activamente en el uso y la generación de conocimiento de innovación y de criticidad. Esta actitud mundana de relacionarse con el Otro y con el mundo por un deseo de la voluntad abre la posibilidad de dialogar e interpretar con una intencionalidad y un significado que el aprendiente posee de la existencia, posibilitando ahondar en los problemas que plantea una inteligencia intuitiva e hipotética. En consecuencia, la primera forma de teoría personal surge de las relaciones con la cotidianidad, como una necesidad de los procesos de socialización. Está dirigida a permitir la interacción social sobre la base de un determinado comportamiento en contextos específicos de acción social. (Medina, 1997, p. 40)

El temor, según Heidegger (2007), es un modo de encontrarse, en cuanto aquello que se teme, se constituye en una amenaza para la existencia por no conocer el espectro de las circunstancias de la vida; la nocividad entendida como los continuos golpes que recibe la persona para formar parte activa de la sociedad, como un ser útil que brinda alternativas de crecimiento para forjar la sociedad donde los sueños se desvanecen por no hacer realidad el proyecto de vida personal por circunstancias endógenas o exógenas que interfieren en el logro de las metas personales; la inseguridad para asumir el futuro de la vida en razón de no poseer la certeza en torno a lo que hace o piensa hacer, generando ansiedad o frustración frente a los retos de la existencia; también, existe la posibilidad de fallar ante los retos que impone la existencia, obligando a la persona a asumir proactivamente la vida u optar por una actitud de indiferencia o negación de su propio ser.

Heidegger (2007) considera que el temer mismo significa tener libertad para dejarse herir. Por tanto, el aprendiente debe interactuar con los otros y con el mundo para permitir que lo hiera en la manera de relacionarse con las demás personas, en las experiencias que obtenga para validar el pensamiento intuitivo e hipotético, en el modo de compartir las emociones y los sentimientos, las frustraciones y el éxito de la vida, el asumir el proyecto de vida personal con el Otro; esto significa hacer gala de la libertad para comprometerse consigo mismo y llegar a una apertura total del ser ahí arrojado en el mudo para deconstruir-construir la realidad y configurar una existencia 
de posibilidades que le proporcione sentido y significado a la existencia a partir de la pregunta y la respuesta. Lo anterior ratifica que

la alquimia del afecto por el saber, la capacidad de generar espacios de ternura y profunda sensibilidad afectiva en su búsqueda y, el placer que nos debe producir el éxito del aprendizaje refrendado por nuestra certeza argumentada de saber que se sabe, constituyen el acto mágico y transformador de un erotismo cognitivo que centra su atención en la permanente gratificación de nuestros logros. (Medina, 1997, p. 51)

El aprendiente piensa que se encuentra en peligro en la vida por aquello por lo cual se teme, según Heidegger (2007): en cuanto asume una interacción de incertidumbre para ser aceptado o rechazado en la sociedad, significa que el peligro se encuentra presente en la vida cotidiana y el mundo de la vida. De ahí surge la duda de lograr un reconocimiento social, de conseguir un empleo, de lograr el éxito laboral, de terminar una carrera, de conseguir los recursos económicos necesarios para mantenerse a sí mismo o mantener la familia, de conseguir una vivienda digna, de tener los bienes necesarios para sobrevivir de educarse y educar a la familia, etc. En el momento en que nace el ser humano, se encuentra en el límite de la vida y la muerte, por ende, debe tratar de encontrarse y de curarse para cerrar el peligro y recobrarse a través de la comunicación y otros recursos existencialistas de orden primario y secundario que le permitan llevar una vida digna en medio del peligro y el abandono.

Partiendo de las premisas de Heidegger (2007), temer por un encontrarse con Otros produce pavor, porque se desconoce la reacción y las respuestas en torno a un diálogo interactivo de sana convivencia. Sin embargo, la realidad muestra que el conflicto está a flor de piel en el hombre, por motivos ideológicos, políticos, sociales, culturales, científicos, religiosos, económicos, de raza y otros, que ponen a los grupos sociales en una zozobra continua de intranquilidad que puede terminar en acciones violentas de aniquilación de la vida. Por tanto, encontrarse con Otro es de riesgo y de peligro, porque no se sabe cuál es la reacción en la calle, en el trabajo, en un sitio público, en el hogar, en la oficina, en el campo, en el transporte, en una estación, etc. Es decir, la persona sale de la casa a la calle, pero con miedo y temor de no saber qué pasará con su vida en el transcurso del día; ello se constituye en una posibilidad existenciaria del ser ahí, no siendo la única para abrirse al mundo.

El aprendiente, al permanecer abierto al mundo, comprende el significado de las cosas. Según Heidegger (2007), puede ser cuando proyecta la existencia mediante acciones de reflexión, de deliberación, de argumentación, de contraargumentación, de toma decisiones, o sea, a través de las habilidades de pensamiento para tratar de problematizar la realidad mediante preguntas y respuestas comprometidas sobre la existencia de sí mismo y de la sociedad. Comprender la realidad es la posibilitad de realizarse de modo diverso ante la propia existencia. Por tanto, el aprendiente comprende que en su propia existencia encuentra el significado de la vida en el campo laboral, familiar, social, político y educativo, porque se abre a sí mismo para transformar esa realidad de acuerdo con los intereses personales inmersos en la cotidianidad y en mundo de la vida.

El aprendiente, cuando se proyecta en torno al proyecto de vida, se abre a la sociedad del conocimiento mediante la profesionalización que le permite ver a través de la estructura 
como ciudadano, como profesional, como padre de familia, como científico, como político, como investigador y como un estudiante que posee una formación integral para afrontar la existencia de sí mismo y de los Otros. Comprender significa cambiar la visión de la vida, porque se apropia de nuevas experiencias y conocimientos según la problematización de la vida, la ciencia, la tecnología y las disciplinas, lo cual conduce al uso y la generación de conocimiento de innovación y de criticidad.

Comprender para el aprendiente requiere partir de un saber determinado, por consiguiente,

la propuesta consiste en que este sea quien desarrolle una dinámica de contrastación permanente con sus propios puntos de vista y que asuma como mecanismo de fortalecimiento de su saber el diálogo argumentado y constante de sus enfoques con los de su maestro y compañeros. Esto requiere no solo del desarrollo de un espíritu investigativo activo y constructor, sino además el desarrollo de habilidades del orden superior que le permitan llegar a sus propias elaboraciones conceptuales, desde las cuales, recupera la voz su discurso argumentador y explicativo. (Medina, 1997, pp. 58 y 59)

La comprensión lleva a la interpretación de los fenómenos del mundo, de acuerdo con Heidegger (2007), se refiere a la estructura del estado expreso de lo comprendido. En otras palabras, cuando el aprendiente comprende algo a partir del conocimiento problematizado es capaz de explicar el conocimiento aprehendido desde la estructura cognitiva que posee para desencadenar nuevos conceptos y teorías emergentes que contribuyen a la configuración de un nuevo pensamiento y vuelve más complejo el esquema de la estructura mental referido siempre a los conocimientos previos adquiridos en el transcurso de la existencia. Entonces, la biografía del aprendiente indica apropiación e intelección de los problemas para expresar de otro modo el conocimiento problematizado según el pensamiento hipotético que tenga de la vida, de la ciencia y la tecnología, la política, la sociedad y la cultura. Esto significa el desarrollo de una inteligencia encarnada que se vuelve más compleja por la percepción sensomotora y la dimensión global de la persona para ver, juzgar y actuar desde una cognición encarnada que tiene conciencia de la situación problematizada.

La interpretación es clave en la problematización del conocimiento, porque requiere pasar de una comprensión cotidiana a un realismo interpretativo,

según el cual el mundo es de una forma determinada, tiene una estructura y características dadas (por ej., la naturaleza discontinua de la materia), pero es imposible acceder directamente a esa estructura, por lo cual es necesario interpretar nuestras impresiones y percepciones a través de ciertos conocimientos o teorías científicas: una especie de "anteojos cognitivos" que nos permitan ver el mundo tal como realmente es. Ciertos rasgos reales del mundo no pueden percibirse directamente en condiciones normales (como los átomos, las células o la corriente eléctrica), pero pueden interpretarse a partir de ciertas experiencias. (Pozo, 2008, p. 464)

Para Heidegger (2007), toda interpretación conlleva la comprensión, porque en la proposición emite un juicio, el cual tiene una finalidad variada y se relaciona con la búsqueda de la verdad. En el análisis de una proposición, presenta tres significados referidos a la indicación, a la predicación y a la comunicación. En la proposición de indicación, se muestra la representación de las cosas en cuanto son, porque en el proceso de la comprensión y la interpretación se construye la representación mental de las cosas y se enuncian como 
son; el aprendiente, desde un realismo de la interpretación existenciaria, debe pasar de un realismo a un idealismo del conocimiento para contar con un repertorio de conceptos más complejos. La proposición predicativa significa conocer el uso y la generación del conocimiento a partir del concepto mismo en torno a lo que expresa y la determinable indeterminación de lo que es; por ejemplo, el martillo tiene el significado de pesantez. La proposición de comunicación significa que el aprendiente se reconoce mediante lo que es común a todos sobre la situación problematizada del conocimiento, afirmando un sentido válido de los actos de habla de lo que es "ser en e mundo", un ser que oye y se comunica con los otros a través del lenguaje verbal para hacer valer el pensamiento y las situaciones problematizadoras del conocimiento, fundado en el valer de la verdad y en la búsqueda de la objetividad del conocimiento.

La interpretación comprensora, vista desde Heidegger (2007), implica ver el entorno con el enfoque de Varela (2000), donde la dimensión local (percepción motora) y la dimensión total (psicología del sujeto) contribuyen al desarrollo de una cognición encarnada, cuyas emociones y cogniciones tienen su origen en la encarnación del conocimiento. Por tanto, la interpretación comprensora parte de una corporeidad que manipula el medio externo, porque tiene el deseo y la voluntad de comprender la realidad a partir de la existencia del ser ahí arrojado en el mundo. En la medida en que el aprendiente manipula las cosas, puede elaborar representaciones mentales de acuerdo con los conocimientos previos adquiridos en el transcurso de la vida. Por ende,

la comprensión implica traducir o asimilar una información nueva a representaciones previas. El aprendizaje no se basa en repetir o reproducir la información presentada como si fuera un hecho dado, sino que requiere activar de forma explícita o deliberada estructuras de conocimiento o representación previas a las que asimilar la nueva información. (Pozo, 2008, p. 190)

La comprensión se estructura a través del logos verbal que emite juicios afirmativos o negativos donde intervienen las habilidades de pensamiento de orden superior para interpretar la realidad referida a un problema que es a la vez una hipótesis. En otras palabras, toda comprensión conlleva la emisión de juicios sobre la realidad existenciaria del ser ahí, constituyéndose al tiempo en posibilidades existenciarias de significado sobre las situaciones problematizadas que tienen implícito un logos del conocimiento. La pregunta y la respuesta es punto de partida de un logos existenciario que encarna una conciencia de sí mismo, del otro, del mundo y de todo lo que lo rodea. En otros términos, la interpretación comprensora alcanza el máximo nivel cuando se encuentra de modo consciente con las posibilidades existenciarias de sobrevivir y proyectar la existencia. Entonces, la conciencia,

ya sea en forma de procesos de control o de reflexión, puede ayudar a los aprendices a dirigir mejor su propio proceso de aprendizaje. ¿Pero cómo pueden acceder a esa conciencia, cómo pueden llegar los aprendices a explicitar sus teorías implícitas para así poder cambiarlas? Sin duda a través de tareas que requieran de ellos un ejercicio sistemático de esos procesos conscientes: planificar, regular la propia práctica y corregir sus errores, evaluar los resultados obtenidos, reflexionar sobre sus formas de aprender y sobre lo que están aprendiendo, etc. Pero todas estas tareas, aun en mayor medida, que otras posibles actividades de aprendizaje, son muy difíciles sin el apoyo o andamiaje externo de los maestros. (Pozo, 2008, p. 369) 
El lenguaje y la palabra posibilitan que el aprendiente se encuentre y comprenda la existencia de sí mismo, de los otros y del mundo respecto del sentido del fundamento de su propio ser ahí. Entonces, para Heidegger (2007), las palabras proveen significaciones de comprensibilidad, porque se refieren al modo de ser en el mundo, en el cual se pone de manifiesto la estructura de las posibilidades de oír y de callar. Por tanto, el aprendiente problematiza el conocimiento, porque tiene la oportunidad de preguntarse y expresarse sobre el mundo para tratar de contrastar o verificar el pensamiento hipotético en el fenómeno de la comunicación, con el fin de coencontrarse y cocomprender la realidad que lo rodea. La única manera de hacerlo es por medio de la palabra para abrir posibilidades existenciarias de afecto, de amor, de diálogo, de compañía, de compartir argumentos y contraargumentos, de establecer lazos de amistad, de compartir experiencias de la vida, de ponerme en el pensamiento del Otro para construir la verdad conjuntamente, de vivir la vida a través de la palabra, siendo esta el grado más alto de inteligencia y de evolución de la humanidad.

Lo que se habla y lo hablado es una notificación de conocimiento existenciario. Por tal razón, la problematización del conocimiento pone en juego las expresiones existenciarias de la formación del espíritu científico en cuanto debe notificar y verificar el funcionamiento de los postulados de la ciencia y la tecnología para formar parte de la sociedad global. Sin embargo, la problematización del conocimiento, no solo se refiere a los postulados científicos, sino que también abarca la vida misma para convertirse en un ser humano digno de convivencia, de respeto, de honestidad y de rectitud. La posibilidad existenciaria de la comunicación es una posibilidad cognitiva y moral. El lenguaje y la palabra adquieren relevancia de humanidad a través de la moralidad, es decir, ser en el mundo existenciario con una notificación de moralidad; de ahí que es conveniente oír y callar con significado de moralidad.

El hombre cuando habla tiene la posibilidad de escuchar al Otro, favoreciendo un diálogo de comprensión; ahora, ello no quita caer en la trivialidad de la conversación. No obstante, toda comunicación se refiere a algo en el mundo y permite la problematización del conocimiento cuando las posibilidades existenciarias de probarlo se agotan, generando preguntas y cuestionamientos que garanticen el mínimo de comprensión de la realidad cuestionada, siendo la palabra el único camino para avanzar en el horizonte del conocimiento.

En los procesos de aprendizaje, la comunicación en el plano existenciario contribuye a la problematización del conocimiento cuando se considera la argumentación un proceso discursivo de entendimiento entre proponentes y oponentes, donde los implicados tematizan una pretensión de validez que se ha vuelto problemática y, exonerados de la presión de la acción y la experiencia, adoptando una actitud hipotética, examinan con razones, y solo con razones, si procede reconocer o no la pretensión defendida por el proponente.

Finalmente, la argumentación puede ser considerada desde un tercer punto de vista: tiene por objeto producir argumentos pertinentes, que convenzan en virtud de sus propiedades intrínsecas, con los cuales desempeñar o rechazar las pretensiones de validez. Los argumentos son los medios con los que puede obtenerse un reconocimiento intersubjetivo para la pretensión de validez que el proponente plantea por de pronto de forma hipotética, y con los que, por tanto, una opinión puede transformarse en saber (Habermas, 2001, p. 47). 
El papel del aprendiente en el plano existenciario tiene sentido en la acción comunicativa cuando los participantes encuentran en el argumento un espacio también para contraargumentar, lo cual posibilita compartir cogniciones encarnadas del conocimiento intersubjetivo, del conocimiento social y del conocimiento científico. Además, la pragmática del acto de habla remite a un diálogo argumentativo sobre los significados que tengan los participantes del mundo diario y del mundo de la vida, el cual problematiza el conocimiento científico desde el ámbito de los imaginarios de la sociedad, de la cultura y de los símbolos. Por tanto, el lenguaje no es estrictamente un instrumento de comunicación que permite compartir las experiencias racionales de la vida, nutriendo el conocimiento con un significado de problemas que afectan al grupo o directamente a la sociedad en el plano del mundo de la vida. De ahí que las razones humanas compiten en el plano existencial por posicionarse con una pretensión de validez argumentativa, porque intentan convencer con razones de consumismo, de arte, de mercantilismo, de religión, de ciencia, de tecnología, de erotismo, de sexualidad; todo aquello que afecta el mundo de la vida del hombre contemporáneo.

Entonces, el significado en el plano existenciario radica en las razones que exponga el hombre para sobrevivir, para compartir momentos de diálogo y de entendimiento, que contribuyan a una sana convivencia en medio de las situaciones conflictivas que alteran el normal desarrollo de la acción comunicativa. El significado de la problematización del conocimiento en torno a la pregunta y la respuesta surge de los problemas de la vida misma que nacen en las interacciones de la pragmática del acto de habla. El hombre intenta responder a una serie de preguntas que lo atormentan acerca del sentido de la propia existencia y las razones para interactuar con otros, con el fin de crear una red de razones que se entrelazan en medio de aspiraciones sociales, culturales, políticas, económicas, científicas, técnicas, tecnológicas, religiosas y otras. Por tanto,

las hipótesis que Habermas tratará de probar es que entender en todo su alcance el fenómeno de la comunicación lingüística no basta con reconstruir el sistema de reglas gramaticales que el hablante debe dominar para formar una oración correcta, sino que tienen que determinarse también las condiciones bajo las cuales esta oración bien formada puede insertarse en un contexto comunicativo y enmarcarse en una realidad determinada (se puede "situar") y, convertida así en un acto de habla, puede aspirar a su "aceptación" por parte de un oyente que considera este acto de habla como válido, en virtud tanto de su contenido proposicional como de las condiciones normativas y contextuales en las que ha sido proferido. (Fabra, 2008, p. 104)

La problematización de la pregunta y la respuesta surge de los problemas del acto de habla en cuanto la forma de habérselas con otras corporeidades que retan la propia existencia de cada individuo. Por tanto, el contenido proposicional depende de la situación en que se encuentren los participantes respecto de la vida misma que le imprimen un significado particular y vivencial para afrontar las circunstancias de acuerdo con la experiencia de la propia biografía del proyecto de vida personal, la biografía familiar, la biografía laboral, la biografía científica y la biografía que tenga de la identidad humana. La pregunta y la respuesta surgen de una experiencia existencial que adquiere el ropaje proposicional de acuerdo con las cogniciones encarnadas y las cogniciones ideales que desea alcanzar para proyectarse en la sociedad. Por otra parte, la problematización del conocimiento tiene que enmarcarse en la dialéctica del argumento y contraargumento si desea involucrarse en las motivaciones e intereses del Otro, con el ánimo de lograr una conceptualización deliberada de los problemas que afectan a la humanidad. 


\section{La pregunta y la respuesta desde la perspectiva de la vida}

El aprendiente se realiza cuando asume un proyecto de vida personal que implica su propia existencia y vitalidad para habérselas con el mundo. Por tal razón, todo el tiempo se la pasa respondiendo preguntas y respuestas para construir una vida social, cultural, científica, económica y política para formar parte de una sociedad que traza un camino permanente y dinámico sobre la base de la sociedad del conocimiento. Entonces, las preguntas y respuestas tienen que ver con su propia biografía para tratar de sobrevivir en un ahora existencia y proyectarse al futuro con un proyecto de vida consolidado sobre sí mismo y en relación con el otro. Esto implica que la persona construya un pensamiento de vida en torno a un proyecto educativo que involucra el conocimiento de sí mismo, de la sociedad de la cual forma parte y del aporte que brinda el conocimiento científico.

Es importante que el aprendiente encuentre un significado de las preguntas que plantea acerca del proyecto de vida, con e fin de problematizar el conocimiento en torno al mundo que lo rodea a partir de un conocimiento encarnado de manipulación de las cosas y propiciar una reflexión personal según una situación dada y las circunstancias que generan la tensión de la relación con los otros para comprender y entender el mundo, con el fin de realizarse plenamente como persona. Por ende,

la vida se me presenta como una pregunta de cuya respuesta depende mi destino. Por una parte, advierto que aún no soy lo que debiera ser; hay una gran distancia entre mi ser potencial o germinal y mi ser en plenitud; es entonces cuando experimento el vacío, la indigencia, la falta de hartura y me siento angustiado, no feliz. Por otra parte, advierto que solo seré feliz cuando logre, por la realización de mi potencial, la plenitud de mi vida. (Sedano, 2002, p. 184)

La realización del aprendiente se encuentra enmarcada en el fenómeno de la vida, puesto que debe responder a las preguntas sobre ¿Cómo habérselas con el mundo según la percepción y los sentidos de la dimensión global de la persona para construir conceptos y teorías que lo lleven a la plenitud de la vida? ¿Cómo perfeccionarse en medio de la imperfección para lograr la máxima realización de sí mismo y del otro? ¿Cómo realizar un proyecto histórico personal de modo consciente para encontrar un camino a su vida? Por consiguiente, el significado de la pregunta y la respuesta existencial conlleva una formación integral de una vida plena, autónoma, deliberada y responsable en concretar el ideal de felicidad.

Sedano (2002) sostiene que, para responder una pregunta autónoma, sin trampas y tapujos, tiene que brotar desde adentro, sin presión alguna que permita tomar alternativas, ya sea de uno u otro lado. El aprendiente debe plantear respuestas que broten de manera sincera y honesta del interior de sí mismo para no engañarse con artimañas que impidan encontrar el camino correcto mediante una decisión deliberada y reflexiva frente a las diversas alternativas por escoger o seleccionar. Por tanto, la pertinencia de las emisiones del aprendiente debe partir de una toma de decisiones que considere la pregunta honesta para brindar una respuesta sincera y autónoma.

Sedano (2002) afirma que la respuesta queda en suspenso y no se realiza un proceso demostrativo para adherirse a cualquier alternativa, debido a que una o la otra alternativa constituye posibilidades reales. Por esta razón, es una pregunta 
dialéctica abierta, dado que se enfrenta a lo imprevisible de lo que piensa la otra persona. De ahí que

matar la palabra - la propia palabra que es la respuesta reveladora de la propia vocación y del propio destino- es matar desde el principio el germen de la responsabilidad como capacidad de respuesta, autónoma y vital, libre y deliberada. (p. 184)

Esto quiere decir que el aprendiente en el transcurso de su vida formula preguntas para encontrar el sentido de la vocación de su propio destino, el cual es el germen de respuestas autónomas, espontáneas y existenciales en torno al fin de su destino. Por ende, las respuestas a las preguntas son progresivas y continuas, persiguiendo siempre la verdad de los acontecimientos, de los sucesos, de las cosas, de las circunstancias y de los problemas de la vida. Las preguntas y las repuestas acerca del proyecto de vida siempre generan ruido, que puede ser un ruido ensordecedor u optar por una actitud de indiferencia. La vocación del estudiante consiste en formular respuestas certeras y verdaderas que no desconozcan el ruido que generan para tratar de construir un proyecto de vida que responda al fin propuesto, sobre sí mismo, los otros y Dios.

El aprendiente debe orientar su vida de acuerdo con unos criterios que surjan de las preguntas vitalistas y existenciales, constituyéndose en el motor para alcanzar los ideales del destino en medio de los ensayos y los errores, con el interés de escribir su propia biografía de vida personal y psicológica, al tiempo que se nutre con las creencias, las costumbres y los comportamientos de la sociedad. Esta impronta vitalista impulsa al aprendiente a formular preguntas y respuestas que nacen de las emociones, de los sentimientos, de los afectos, de los valores y de una inteligencia encarnada que abarca la dimensión global de la persona, irrumpiendo con acciones buenas o malas para sí mismo y con los otros. Ahora, Sedano (2002) concibe el concepto de bueno como la plenitud del ser como existencia radical; en caso de no lograrlo, será un ser bueno relativamente. En consecuencia, "a mayor realización de su existencia, mayor perfección, de modo que será totalmente perfecto por llegar a plenitud de la vida, que le es propia" ( $p$. 198).

Las preguntas y las repuestas llevan a una actualización del aprendiente en torno a los intereses de sí mismo, mirando las capacidades de orden biológico, psicológico, cognitivo, social, cultural, científico, vital, existencial y trascendental. Estas preguntas brotan de sí mismo para encontrar respuestas en medio del misterio y de la incertidumbre de la vida, con el firme propósito de realizarse por sí mismo, porque se siente movido en hacerlo. "En la medida en que se realiza a sí mismo por sí mismo, se perfecciona como persona humana; y en esta proporción está haciendo su propio ser hacia la plenitud de su ser" (Sedano, 2002, p. 199).

La pregunta y la respuesta contribuyen a la realización del aprendiente cuando se afirma en una convicción de responsabilidad de sí mismo, con el fin de que toda acción persiga el sentido del bien para educarse a plenitud en torno al proyecto de vida personal, ya sea de orden familiar, laboral, social, comunitario y político. Esto quiere decir que la pregunta y la respuesta nace de una convicción ética y axiológica donde todo aquello que es bueno afecta el accionar de la vida. Cuando se aparta de la bondad de la vida, se niega a sí mismo y al Otro, dado que pone obstáculos para realizarse en medio de los cuestionamientos que genera la existencia para sobrevivir y el mantener la vitalidad para relacionarse con otras corporeidades que retan el ser ahí arrojado en el mundo. “Esta es precisamente la densidad y riqueza del 'bien' y de 'lo 
bueno', definido en la perspectiva de la plenitud de realización Humana" (Sedano, 2002, p. 201).

El aprendiente forma el juicio en el transcurso de la vida, lo cual implica ver, juzgar y actuar para orientarse a sí mismo y orientar a los demás con un sentido de mayoría de edad de criticidad y de prudencia, con el interés de tomar decisiones responsables y deliberadas que favorezcan una buena construcción asertiva de la libertad. Entonces, el aprendiente asume la responsabilidad de las acciones y no se le achacan a los demás, por tanto, las preguntas y las respuestas que se formulen están en las manos del aprendiente para autodeterminarse, autodecidirse y autoobligarse; en otros términos, la pregunta y la respuesta posibilitan formar el criterio del aprendiente. De ahí que, "en la precisa medida que vayamos creciendo en juicio y en autodecisión hacia la adultez, nos vamos guiando nosotros mismos por nosotros mismos" (Sedano, 2002, p. 202).

\section{La pregunta y la repuesta como instancia de educarse a sí mismo}

La pregunta y la respuesta para educarse a sí mismo radica en la confianza que tenga el individuo para asumir el proyecto de vida personal. Por consiguiente, el aprendiente tendrá que dar cuenta del manejo responsable de la propia libertad para asumir la personalización de educarse a sí mismo, tomando la iniciativa de la vida en forma creativa para explorar su propio significado de autenticidad en beneficio del bien común. Por ende, el estudiante debe educarse a sí mismo mediante el potencial cognitivo y metacognitivo, las emociones, los sentimientos, los afectos, la inteligencia encarnada, la mente y el cuerpo. Esto implica que tiene que moverse por sí mismo para alcanzar la problematización del conocimiento en cuanto debe tomar la iniciativa para explorar por sí mismo el mundo comprometiéndose a hacer realidad las respuestas en torno a todo lo que cuestiona para conocer el mundo, pensar en torno a la cuestión, deliberar sobre los problemas de la cuestión, sintetizar el conocimiento y validar las posturas del pensamiento hipotético.

Sedano (2002) sostiene que no se puede personalizar sin definirse, entendiendo que la persona debe moverse para lograr la realización personal. De ahí que debe darse salida a la creatividad, a la inventiva, al ingenio, a las ideas, en fin, a los proyectos personales para definirlos en el quehacer diario de la vida. Entonces, el individuo deberá responsabilizarse de las respuestas en la medida en que toma decisiones para proyectarse en la vida.

En definitiva, nos deFINimos, no por condeFINarnos en los estrechos límites de una categoría o un concepto explicativo de nuestra naturaleza humana, sino por ponernos en marcha hacia un FIN; no cualquier FIN, sino ese en el cual hemos fincado nuestro propio destino. (p. 213)

La personalización hacia un fin implica que la persona tome las mejores decisiones para llegar a la mayoría de edad, lo cual significa tomar decisiones prudentes y críticas a fin de forjar una personalidad prudente que lo mueva a ser una mejor persona en búsqueda del bien común.

También Sedano (2002) afirma que la persona no puede definirse sin autodeterminarse, cuando pasa de la heteronomía a la autonomía. En otras palabras, la persona tiene que deliberar y establecer las razones para obrar a conciencia y 
a voluntad por cuenta propia. La autonomía se construye mediante el diálogo y el conversatorio para aprender a tomar decisiones asertivas y certeras a través de las contradicciones, las semejanzas, las diferencias, la duda, el escepticismo, la inquietud y la curiosidad. Esto quiere decir que la persona se autodetermina cuando mantiene la motivación en medio de las dificultades que surgen en la problematización de conocimiento y las dificultades de la vida, tratando siempre de moverse por sí mismo en torno a lo conocido que lo conduzca a una formación integral como ciudadano y hombre valioso en la sociedad. Por ello,

cuando el hombre actúa por sí mismo hacia el fin, conoce ese fin; pero cuando es actuado o conducido, obrando, por ejemplo, bajo imposición o impulso de otro, no tiene para qué conocer el

fin. Es esto lo que acaece a las criaturas irracionales. (p. 218)

La personalización no tiene sentido si la persona no se compromete consigo misma para la realización del proyecto de vida personal donde se involucra a las otras personas a fin de que asuman de manera responsable las acciones de la vida diaria y del mundo de la vida. De ahí que

aprendemos a respondiendo. Y responder es, en primer término, comprometerse con la realidad ambiente en la que estamos ya embarcados, previa aceptación consciente de nuestra condición encarnada en situación concreta; responder es abrirse a la realidad de nuestro mundo para aprender de él o discernir en vida la verdad, en nuestro caso, la verdad definidora de nuestra vida. (Sedano, 2002, p. 221)

El compromiso se reafirma mediante la comunión que tenga el aprendiente para formular respuestas a fin de comprometerse con el Otro en el plano existencial en búsqueda de repuestas de convencía, de solidaridad, de amistad, de problematización del conocimiento, de reconocimiento de la dignidad de la persona humana, de amor y compromiso frente a una sociedad caótica y que no fomenta una coexistencia pacífica de afirmación de una identidad de comunión de vida amorosa.

Decidirse es el acto central de la libertad, porque es la clave de la autonomía al tomar decisiones deliberadas y de juicios críticos para llevar a cabo acciones justas, equitativas, solidarias y de beneficio al bien común. Cuando un aprendiente se decide a problematizar el conocimiento, busca la verdad, por ende, está sustentada en la convicción, en la experiencia, en el reconocimiento del aporte argumentativo del Otro, en la veracidad y la honestidad de lo que se dice, en la sinceridad, en la intelección y comprensión e interpretación de los problemas, en la verificación de las respuestas, en el aprender a escuchar en el diálogo, en conocer diferentes puntos de vista, en analizar las situaciones, en tomar decisiones que enriquezcan el proyecto de vida personal para ver, juzgar y actuar con prudencia.

Decidirse por convicción implica que la persona está plenamente comprometida de modo creativo en el proyecto de vida personal. Por esta razón, la convicción propicia una inventiva muy proactiva para el cumplimento de las metas personales, porque el individuo está convencido de sortear todos los peligros de la vida con una motivación intrínseca y una actitud tan positiva, en la que puede ser frustrante la experiencia, con el fin de aprender de los errores como fuente de nuevos conocimientos problematizados alrededor de la justicia, de la paz, de la armonía, de la convivencia, el respeto y el amor al otro. "Por convicción. Se trata de un juicio, una verdadera autodeterminación personal que brote de mí mismo por mí mismo, ya sea por propio aprendizaje ("Disciplina") o por inventiva o creatividad ("inventio")" (Sedano, 2002, p. 
247). En otras palabras, la autonomía requiere confrontación argumentativa para formar los propios juicios personales respecto de la igualdad y justicia para todos, pero sin caer en un servilismo que atente contra la formación de un juicio prudente.

Las preguntas y las respuestas en la problematización de conocimiento deben contener valores relacionados con la vida. En otras palabras, las preguntas deben encarnar valores que promuevan un desarrollo afectivo, social, cultural y científico, en el sentido de fomentar preguntas y respuestas que estimulen un diálogo de encarnación dirigido a rescatar la dignidad de la persona para favorecer un aprendizaje problematizador agradable y feliz que facilite un aprendizaje significativo. Efectivamente, cuando el individuo se apropia de los valores, "vivimos a partir de ellos, para ellos y por ellos nos dejamos guiar, ya que constituyen, el horizonte existencial de nuestro ser y quehacer: Dime qué amas y te diré quién eres y como vives" (Sedano, 2002, p. 252). Lo anterior quiere decir que, cuando el aprendiz internaliza los valores en las preguntas y las respuestas, favorece las interrelaciones interpersonales el diálogo, la escucha, el debate, el aporte del otro, e reconocimiento a la diferencia, el disenso, el consenso y el "lenguajear" fluido.

La pregunta y la respuesta basada en la fidelidad implican comprometerse sin titubeo en el proyecto de vida personal, ser fiel a los valores, a los compromisos, a las metas de aprendizaje, al ideal de trabajo con los compañeros, ser fiel consigo mismo y con el otro para forjar una respuesta realmente comprometida y personalizada y lograr promoverse a sí mismo como una persona digna con valores de lealtad. Es decir que el docente "debe mirar a formar personas que construyen la propia vida sobre valores seguros - absolutos y transcendentes- y que no se contenten vivir en una mediocridad banal" (Sastoque, 2001, p. 82). La pregunta y la respuesta forma para que los aprendientes construyan una vida arraigada en el respeto de la persona y la vida misma, con el fin de superar las racionalidades instrumentales de cálculos y fines que conducen al hombre a una vida secular donde la persona se pierde en el delirio de las racionalidades e irracionalidades de los discursos que intentan vender antivalores con la etiqueta de humanidad y de eticidad, perdiéndose en un hombre mediocre que discurre en la relatividad de la humanidad.

\section{Conclusiones}

La pregunta y la respuesta es inherente a la curiosidad del hombre, lo cual significa asumir la autorrealización en torno a los dilemas de la vida que generan tristeza, alegría, esperanza, amor y desamor, todo enmarcado en la corporeidad de comprenderse e interpretarse en medio de la problematización del conocimiento, de los afectos, de las emociones, de los sentimientos y de la existencia. Sin embargo, la vida buena significa hacer de la muerte un sentido de vida, de la violencia una no violencia, del conflicto un gran espacio de amistad, de la agresividad lazos de afectividad, lo cual implica crear un repertorio cognitivo de inteligencia encarnada para comprender la complejidad y lo absurdo del hombre contemporáneo. La pregunta y la respuesta es la huella de la inteligencia, de la sabiduría, de los problemas de la sociedad del conocimiento y de la biografía que se escribe en el andar de la vida diaria para preguntarse y responderse, sin tener un límite al interrogatorio. 
La biografía de la vida buena reta a un aprendizaje significativo para entender que los valores no son nominales sino una vivencia de una moralidad que debe buscar el bien común para propiciar espacios de diálogo de preguntas y repuestas cognitivas, metacognitivas, problematizadoras, amorosas, experimentales, sociales, culturales y científicas, y todo aquello que concierne al mundo de la vida. Entonces, la pregunta y la respuesta es desaprender para aprender a escribir continuamente la biografía personal con la biografía del otro y la biografía de la sociedad para ahondar en la profundidad de las preguntas y las respuestas, las cuales remiten al círculo hermenéutico de la existencia donde el hombre trata de habérselas con la realidad, con el mundo y con la sociedad como tal.

\section{Referencias}

Fabra, P. (2008). Habermas: lenguaje, razón y verdad. Los fundamentos del cognitivismo en Jürgen Habermas. Madrid: Marcial Pons.

Habermas, J. (2001). Teoría de la acción comunicativa: complementos y estudios previos (trad. M. Jiménez Redondo). Madrid: Cátedra.

Heidegger, M. (2007). El ser y el tiempo. México: Fondo de Cultura Económica.

Medina, C. (1997). La enseñanza problémica. Bogotá: Rodríguez Quito Editores.

Pozo, J. I. (2008). Aprendices y maestros: la psicología cognitiva del aprendizaje. Madrid: Alianza.
Sastoque Poveda, L. F. (2001). Lineamientos para una identidad del educador dominicano. Bogotá: Universidad Santo Tomás.

Sedano, J. de J. (2002). Pedagogía de la respuesta. Bucaramanga, Colombia: Universidad Santo Tomás.

Varela, F. (2000). El fenómeno de la vida: Cuatro pautas para el futuro de las ciencias cognitivas. Santiago de Chile: Dolmen. 